When do fluid membranes engulf sticky colloids?

This article has been downloaded from IOPscience. Please scroll down to see the full text article.

2004 J. Phys.: Condens. Matter 16 S2061

(http://iopscience.iop.org/0953-8984/16/22/004)

View the table of contents for this issue, or go to the journal homepage for more

Download details:

IP Address: 128.2.23.90

The article was downloaded on 08/03/2012 at 21:15

Please note that terms and conditions apply. 


\title{
When do fluid membranes engulf sticky colloids?
}

\author{
Markus Deserno \\ Max-Planck-Institut für Polymerforschung, Ackermannweg 10, 55128 Mainz, Germany \\ E-mail: deserno@mpip-mainz.mpg.de
}

Received 26 September 2003

Published 21 May 2004

Online at stacks.iop.org/JPhysCM/16/S2061

DOI: $10.1088 / 0953-8984 / 16 / 22 / 004$

\begin{abstract}
Binding of a spherical colloid to a fluid membrane, which is an interplay between the energies of adhesion and elastic deformation, is studied within the framework of a Helfrich Hamiltonian. The solution of the full nonlinear shape equations for the membrane profile reveals a continuous binding and a discontinuous envelopment transition, the latter with a tension dependent substantial energy barrier. In the bending dominated regime this scenario is analytically confirmed by a small gradient expansion.
\end{abstract}

\section{Introduction}

The adsorption of micro- or mesoscopic particles onto soft surfaces provides the possibilityabsent in the case of rigid substrates - that the degrees of freedom of the surface feature prominently in the interaction. A particularly important example for such surfaces is lipid bilayers, mostly of course due to their ubiquity in biology, and one often wishes to study their interactions with for instance proteins in a well controlled set-up-without losing fluidity and local elasticity. This has, among other things, led to the development of supported membranes, separated from the solid substrate by a thin water layer or by a soft polymer cushion [1].

However, for objects which are still small but no longer comparable in size to the membrane thickness, a different surface response will become a likely participant in the adhesion balance: out-of-plane bending. Clearly, such a deformation, which is a prerequisite for any adhesion to a curved object, will entail a bending energy penalty which opposes the original binding. This effect will be significant if only the object's curvature is sufficiently high-for typical cell membrane parameters radii of curvature of a few tens up to a few hundred nanometres will be in the right range. This is unfortunately below optical resolution, explaining why systematic studies of such membrane deformations are difficult.

There is one prominent biological example where such adsorption events have been studied quite carefully: viral budding, the process by which many enveloped animal viruses leave their host cell [2]. After entering the cell (typically via receptor mediated endocytosis or other active processes [3]) and completion of the viral replication steps, the new virions leave their host by 


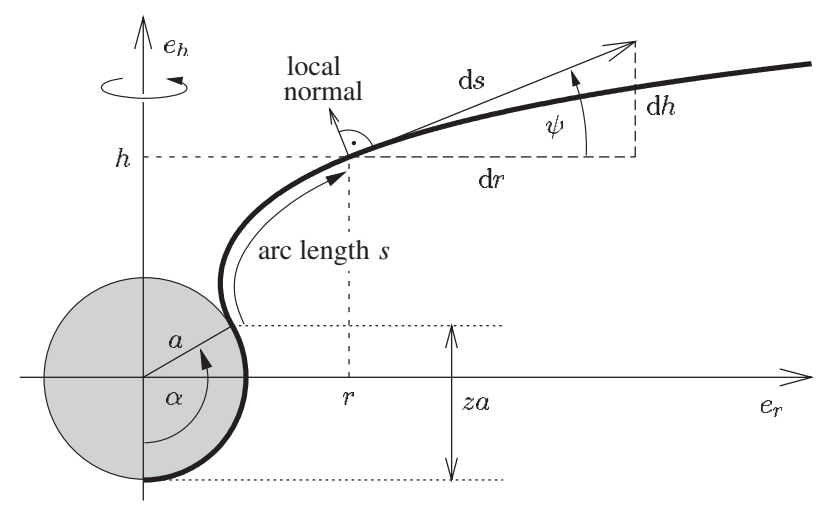

Figure 1. A spherical colloid adheres partially and cylindrically symmetric to a membrane with degree of penetration $z=1-\cos \alpha$. Due to the possibility of overhangs, the profile is described by specifying the angle $\psi$ with respect to the horizontal as a function of arc length $s$.

having their nucleoprotein capsid wrapped by the plasma membrane (or some other membrane of the secretory pathway). This not only sets them free, they also acquire their final coating. A particularly well studied model case is provided by Semliki forest virus, which utilizes viral ('spike'-) proteins to mediate binding between capsid and membrane [4-8].

Many other realizations of such adhesion and wrapping events exist. Boussif et al [9] recently proposed an efficient gene transfection system, in which DNA is complexed by the cationic branched polymer polyethylenimine into a globular complex, which then enters the cell by an adhesion driven invagination process. Moreover, many biophysical experiments require the adhesion of microbeads to cells. For instance, cell tension is routinely probed by measuring the force necessary to pull a thin membrane tether [10]; and the centripetal membrane motion, relevant for cell locomotion, is visualized by imaging adhering marker beads [11]. These are actually engulfed by the cell without involvement of the endocytosis machinery, provided the membrane tension is low enough [12].

This paper presents a theoretical study of the generic features of such processes, in which adhesion of a colloid to a fluid membrane results in a nontrivial binding balance. The main ingredient is the Helfrich Hamiltonian [13], which thus identifies the stage for all that follows as the regime in which continuum elasticity theory is applicable.

\section{Definition of the model}

\subsection{Relevant variables and energetics}

The purpose of this paper is to study the local deformation of a fluid membrane occurring after binding to a spherical colloid. The situation is fully specified by the following variables (see figure 1): the membrane is characterized by a bending stiffness $\kappa$ [13], zero spontaneous curvature, and an applied lateral tension $\sigma$ (which in the biological situation is adjusted by the cell [14]). The combination $\lambda:=\sqrt{\kappa / \sigma}$ then defines a length scale intrinsic to the membrane. Taking typical values of the bending stiffness ( $\kappa \simeq 20 k_{\mathrm{B}} T$ [15]) and lateral tension ( $\sigma \simeq 0.02 \mathrm{dyn} \mathrm{cm}^{-1}$ [14]) we find $\lambda \simeq 64 \mathrm{~nm}$. Next, the colloid has radius $a$ and adheres with an energy $w$ per unit area to the membrane. The amount of wrapping can be specified either by the detachment angle $\alpha$, or more conveniently by the expression $z=1-\cos \alpha$, which we term the degree of penetration, and which varies between zero (just touching) and two (fully 
enveloped). Since $\kappa$ and $a$ provide a natural energy and length scale, respectively, we define the following dimensionless variables:

$$
\tilde{E}:=\frac{E}{\pi \kappa}, \quad \tilde{w}:=\frac{2 w a^{2}}{\kappa}, \quad \tilde{\sigma}:=\frac{\sigma a^{2}}{\kappa}=\left(\frac{a}{\lambda}\right)^{2} .
$$

The size of typical viral capsids is a few tens of nanometres, hence viral budding sits squarely in the crossover regime in which $a \sim \lambda$ and thus $\tilde{\sigma} \sim 1$. In terms of these variables, the energy of a wrapping complex can be expressed as

$$
\tilde{E}=-(\tilde{w}-4) z+\tilde{\sigma} z^{2}+\tilde{E}_{\text {free }}(z, \tilde{\sigma})
$$

where $\tilde{E}_{\text {free }}=E_{\text {free }} / \pi \kappa$ is the rescaled energy of the free part of the membrane. Its determination is the tricky bit of the problem, because the membrane shape first has to be found by minimizing the deformation energy (see below). However, neglecting it in a first crude approximation immediately yields the result that colloids do not adhere if $\tilde{w}<4$ and become fully enveloped once $\tilde{w}>4+4 \tilde{\sigma}$. In between they adhere partially with a degree of penetration given by $z=(\tilde{w}-4) / 2 \tilde{\sigma}$. For $\sigma=0$ this agrees with the earlier predictions in $[16,17]$.

\subsection{Shape equations for the free membrane}

The energy of the free membrane is a functional of its shape. Within the parametrization of figure 1 the two local principal curvatures are found to be $\dot{\psi}$ and $(\sin \psi) / r$, the dot indicating a derivative with respect to the arc length $s$. The energy functional can then be written as $\tilde{E}_{\text {free }}=\int_{0}^{\infty} \mathrm{d} s L$, where the Lagrangian $L$ is given by

$L=r\left[\left(\dot{\psi}+\frac{\sin \psi}{r}\right)^{2}+\frac{2 \tilde{\sigma}}{a^{2}}(1-\cos \psi)\right]+\lambda_{r}(\dot{r}-\cos \psi)+\lambda_{h}(\dot{h}-\sin \psi)$.

The terms in square brackets are the curvature and tension energies, respectively, while the remaining two expressions fix the nonholonomic parametrization constraints $\dot{r}=\cos \psi$ and $\dot{h}=\sin \psi$ by means of the Lagrange parameter functions $\lambda_{r}$ and $\lambda_{h}$. The momenta canonically conjugate to $\psi, r$ and $h$ are

$p_{\psi}:=\frac{\partial L}{\partial \dot{\psi}}=2 r\left(\dot{\psi}+\frac{\sin \psi}{r}\right), \quad p_{r}:=\frac{\partial L}{\partial \dot{r}}=\lambda_{r}, \quad p_{h}:=\frac{\partial L}{\partial \dot{h}}=\lambda_{h}$,

and thus the Hamiltonian is given by

$$
\begin{aligned}
H & :=\dot{\psi} p_{\psi}+\dot{r} p_{r}+\dot{h} p_{h}-L \\
& =\frac{p_{\psi}^{2}}{4 r}-p_{\psi} \frac{\sin \psi}{r}-\frac{2 \tilde{\sigma} r}{a^{2}}(1-\cos \psi)+p_{r} \cos \psi+p_{h} \sin \psi
\end{aligned}
$$

Since the Lagrangian does not depend explicitly on arc length $s$, the Hamiltonian is conserved. Finally, the shape equations for the membrane are the associated Hamilton equations:

$$
\begin{aligned}
\dot{\psi} & =\frac{p_{\psi}}{2 r}-\frac{\sin \psi}{r} \\
\dot{r} & =\cos \psi \\
\dot{h} & =\sin \psi \\
\dot{p}_{\psi} & =\left(\frac{p_{\psi}}{r}-p_{h}\right) \cos \psi+\left(\frac{2 \tilde{\sigma} r}{a^{2}}+p_{r}\right) \sin \psi \\
\dot{p}_{r} & =\frac{p_{\psi}}{r}\left(\frac{p_{\psi}}{4 r}-\frac{\sin \psi}{r}\right)+\frac{2 \tilde{\sigma}}{a^{2}}(1-\cos \psi) \\
\dot{p}_{h} & =0 .
\end{aligned}
$$




\subsection{Boundary conditions}

The shape equations $(6 a)-(6 f)$ have to be solved subject to appropriate boundary conditions. At contact, $s=0$, the membrane must smoothly touch the colloid, implying

$$
r(0)=a \sin \alpha, \quad h(0)=-a \cos \alpha, \quad \psi(0)=\alpha .
$$

If the membrane is free to choose the point of detachment, a further condition on the contact curvature applies $[18,19]$ :

$$
a \dot{\psi}(0)=1-\sqrt{\tilde{w}} .
$$

However, since we will be interested in the profile energy at any given degree of penetration, the above condition cannot be enforced. It will later emerge to hold for equilibrium profiles, where $\partial E / \partial z=0$ (see the appendix in [20]). Still, the fact that the equilibrium point of detachment has to be determined self-consistently is one of the tricky aspects of the problem.

The notion of asymptotic flatness will be taken care of by requiring

$$
\lim _{s \rightarrow \infty} \psi(s)=0, \quad \lim _{s \rightarrow \infty} \dot{\psi}(s)=0,
$$

which in particular implies that curvature and tension energy density vanish as one moves away from the wrapping site. Note that we do not require the slightly stronger condition that $h$ should approach a constant.

If $\psi(s)$ vanishes sufficiently rapidly (which an asymptotic analysis of the shape equation confirms, see (13) in section 4), energetic contributions beyond some large cut-off arclength $S$ are negligible. We may thus impose (9) at $s=S$, such that variations of $S$ and $\psi(S)$ are no longer allowed. However, $r(S)$ and $h(S)$ are still free and give the boundary conditions [21]

$$
0=\left.\frac{\partial L}{\partial \dot{r}}\right|_{s=S}=p_{r}(S), \quad 0=\left.\frac{\partial L}{\partial \dot{h}}\right|_{s=S}=p_{h}(S) .
$$

Together with $(6 f)$ the second condition shows that $p_{h} \equiv 0$, so this variable entirely drops out of the problem. The first equation can be turned into a condition at contact by making use of the constancy of the Hamiltonian and the flat profile requirement, which together imply $H=H(S) \stackrel{S \rightarrow \infty}{\longrightarrow} p_{r}(S)=0$. Solving $H=0$ for $p_{r}(0)$ then yields

$$
\operatorname{ap}_{r}(0)=\frac{\sqrt{z(2-z)}}{1-z}\left[1+2 \tilde{\sigma} z-(\operatorname{a\dot {\psi }}(0))^{2}\right] .
$$

The only remaining unknown contact variable is $\dot{\psi}(0)$. For given values of $\tilde{\sigma}$ and $z$ the shape equations now have to be integrated (e.g. by using a shooting method) and the value(s) of $\dot{\psi}(0)$ have to be found which result in an asymptotically flat profile. This basically comes down to a nonlinear eigenvalue problem. Its delicacy is illustrated in figure 2.

\section{Numerical results}

Numerically determining the equilibrium membrane shape and inserting it back into the energy functional yields the energy $E(z)$ of the complex as a function of the degree of penetration. This provides knowledge about the equilibrium structure of the complex as well as possible energy barriers. 


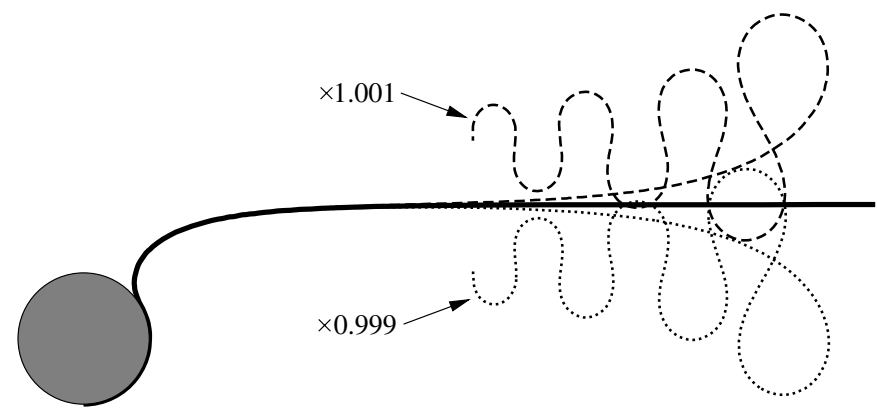

Figure 2. The solid curve shows the membrane profile for a detachment angle of $120^{\circ}$ and an applied tension of $\tilde{\sigma}=0.5$. This requires a contact curvature of about $a \dot{\psi}(0)=-1.18749057$. The dashed and the dotted curves show how the calculation deviates from the flat profile if this contact curvature is off by a factor of 1.001 or 0.999 , respectively.

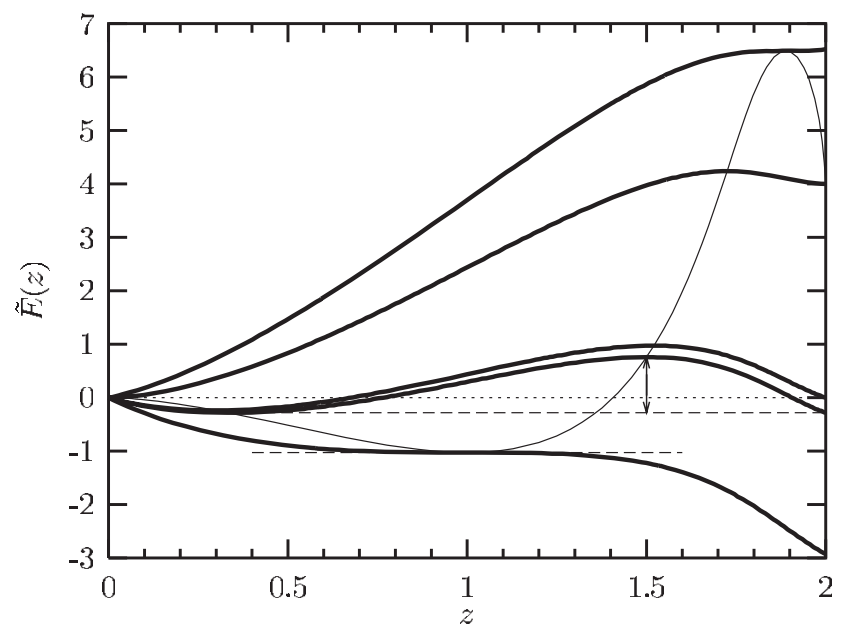

Figure 3. Energy $\tilde{E}(z)$ as a function of penetration $z$ for a rescaled tension $\tilde{\sigma}=1$. From top to bottom the rescaled adhesion energy takes the values $\tilde{w}=2.738$ (upper spinodal), 4 (onset of binding), $6(4+2 \tilde{\sigma}$, enveloped state has zero energy), 6.141983 (envelopment transition) and 7.46410 (lower spinodal). Increasing $\tilde{w}$ will first lead to a smooth binding transition, followed by a discontinuous envelopment transition. Its energy barrier is indicated by the double arrow. The fine solid curve is the locus of all extrema.

\subsection{Equilibrium structure}

Figure 3 shows an example of $\tilde{E}(z)$ as a function of $z$ for successively larger values of the adhesion strength $\tilde{w}$. The minimum ${ }^{1}$ identifies the equilibrium state. At $\tilde{w}=4$ partial binding sets in, which, since the condition is independent of $\tilde{\sigma}$, results from a balance between adhesion and curvature alone. Indeed, (2) shows that this balance takes place one order lower in $z$ than the tension contribution, which enters quadratically (for $\tilde{E}_{\text {free }}$ this follows from a small gradient expansion; see (15) in section 4). Further increasing $\tilde{w}$ will lower the energy of the fully enveloped state until it reaches the energy of the free state. Since in the limit $z \rightarrow 0$ we also have $\tilde{E}_{\text {free }}(z) \rightarrow 0$ (ideal necks have no energy [22]), the point at which $\tilde{E}(z=2)=\tilde{E}(z=0)$

1 The contact curvature condition (8) follows from $\partial \tilde{E} / \partial z=0$. However, this is also satisfied at maxima, and it is not satisfied at boundary minima, at which the slope need not vanish. 


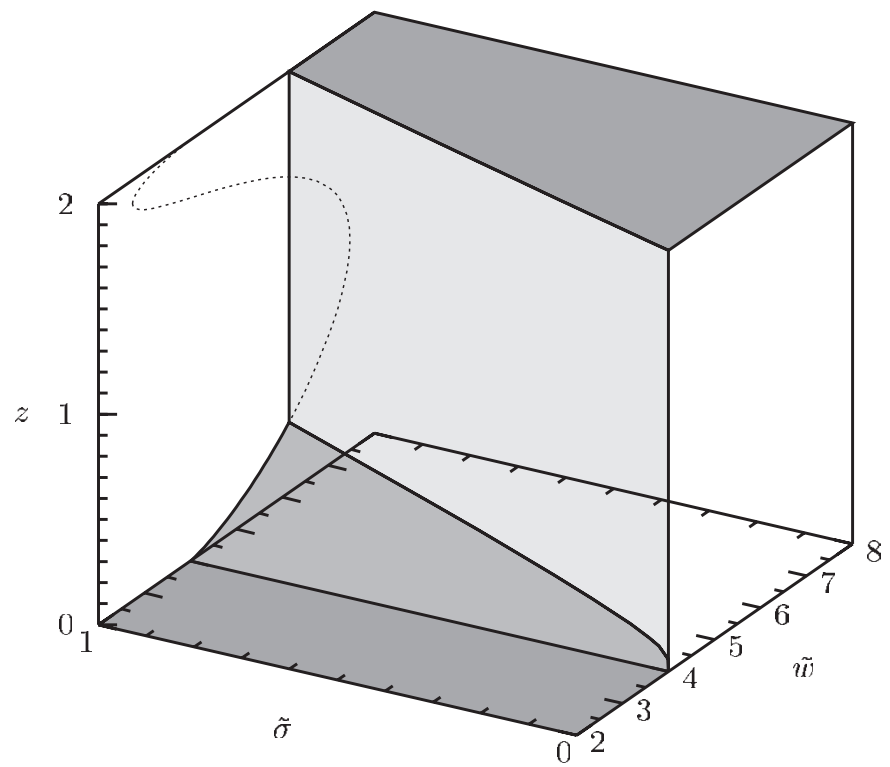

Figure 4. Phase diagram of the wrapping complex in the small tension regime $\tilde{\sigma} \leqslant 1$. The smooth transition from free to partially wrapped (at $\tilde{w}=4)$ can be seen, as well as the strongly discontinuous envelopment transition (roughly at $\tilde{w}=4+2 \tilde{\sigma})$. Both lines meet at $(\tilde{w}=4, \tilde{\sigma}=0$ ) at which the discontinuous jump in $z$ is maximal, but at which also the energy barrier vanishes. At $\tilde{\sigma}=1$ the 'van der Waals loop' is indicated as a dotted curve. At that point, and for $\kappa=20 k_{\mathrm{B}} T$, the barrier height would be about $66 k_{\mathrm{B}} T$, showing that the system will locally rather follow the metastable branches and thus display hysteresis.

is readily seen to occur at $\tilde{w}=4+2 \tilde{\sigma}$. For slightly larger values of $\tilde{w}$ the energy of the fully enveloped state drops below the shallow equilibrium low $z$ minimum. This behaviour is depicted for the low tension case in the structural phase diagram of figure 4.

\subsection{Envelopment barrier}

The jump to the enveloped state is not only discontinuous in $z$, it also requires an energy barrier $\Delta \tilde{E}$ to be overcome (see figures 3 and 5). At $\tilde{\sigma}=1$ its value is $\Delta \tilde{E} \simeq 1$, which for $\kappa=20 k_{\mathrm{B}} T$ yields $\Delta E \simeq 66 k_{\mathrm{B}} T$. This is large compared to thermal energy, hence the system will not readily change its structure at the transition point but rather display hysteresis and follow the metastable wrapping and unwrapping branches. The concomitant "van der Waals loop' is indicated for the case $\tilde{\sigma}=1$ in figure 4 .

For small $\tilde{\sigma}$ the energy barrier can be empirically fitted by $\Delta \tilde{E}_{<}(\tilde{\sigma})=1.54 \tilde{\sigma}^{0.86}$, while for large $\tilde{\sigma}$ it will asymptotically reach the expression $\Delta \tilde{E}_{>}(\tilde{\sigma}) \simeq 3.5 \tilde{\sigma}^{1 / 3} .^{2}$ In the latter case the exponent $1 / 3$ can be derived by a scaling argument [20]. Figure 5 also indicates that this change in exponent between the low tension and high tension regimes finds its counterpart in the jump-size $\Delta z$ : at low tension the jump is large, while at large tension it is small, the crossover coinciding with the crossover of the energy exponent. For large $\tilde{\sigma}$ one has $\Delta z \simeq 3.17 \tilde{\sigma}^{-1 / 3}$, with the exponent $-1 / 3$ again following from scaling [20].

The deeper reason for the existence of an energy barrier is the fact that partially wrapped states deform the membrane more strongly than somewhat wrapped or fully wrapped states. They thus store a substantial elastic energy in the free membrane which impedes the

2 The expression $\left[\Delta \tilde{E}_{<}^{-1}+\Delta \tilde{E}_{>}^{-1}\right]^{-1}$ approximates $\Delta \tilde{E}$ for all $\tilde{\sigma}$ with about $10 \%$ accuracy. 


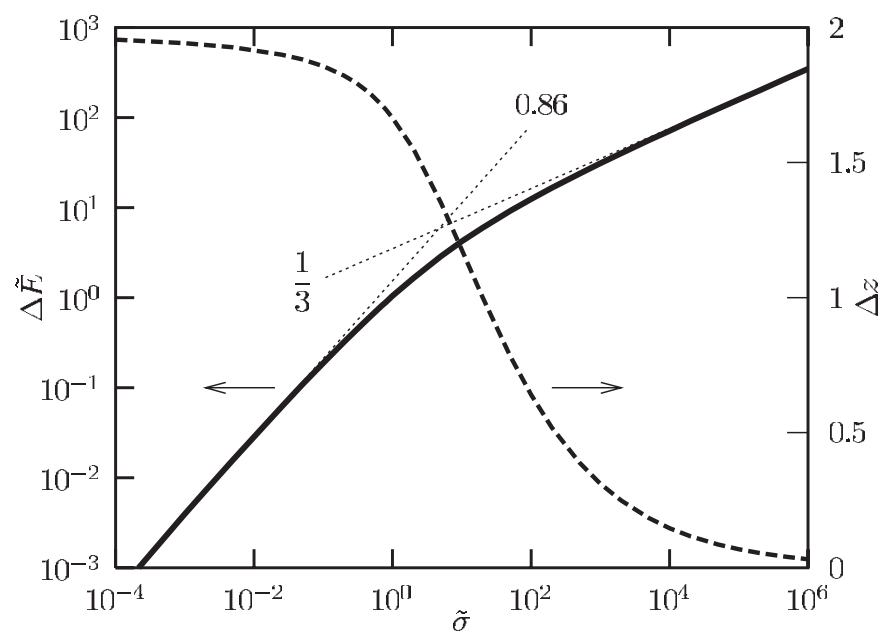

Figure 5. Log-log plot of the barrier height $\Delta \tilde{E}$ as a function of rescaled tension $\tilde{\sigma}$ (solid curve, left vertical scale). The two dotted lines indicate the asymptotic power law exponents. The dashed curve (right scale) shows the corresponding jump in $z$. The crossover in the exponent of the energy scaling is reflected in a change from a large jump $\Delta z$ at small tension to a small jump at large tension.

envelopment transition. However, the situation is a bit more subtle. Let us define the total penetration $d$ as the vertical distance between the asymptotic membrane and the lowest point of the colloid (i.e., how deep the colloid penetrates on the other side with respect to the membrane plane). Figure 6 shows this total penetration $d$ in units of the colloid radius $a$ as a function of the usual penetration $z$ for various membrane tensions $\tilde{\sigma}$. Several observations are noteworthy.

- For any given value of the (usual) penetration $z$ the total penetration $d$ is bigger if the applied tension is lower. This holds because high tensions pull the membrane flat and prevent a large total penetration.

- For low tension the transition point for envelopment occurs at rather low values of $z$ or $d$, as has previously been seen in figures 3 and 4 .

- The (lower) spinodal line, at which the (wrapping) barrier vanishes, occurs at significantly larger penetrations. However, it does not correspond to the maximum in $d(z)$, at which the total penetration (and thus the global deformation) is largest.

- The position of the maximum in $d(z)$ coincides with the nontrivial value of $z$ at which the equilibrium contact curvature $a \dot{\psi}(0)=-1$. This establishes a remarkably simple link between a local property (contact curvature) and the global shape of the profile. Due to the lack of an analytical solution the origin for this is as yet obscure, but it may be related to the following observation: from the contact curvature condition (8) and the fact that binding only sets in for $\tilde{w} \geqslant 4$ it follows that the equilibrium contact curvature can be $a t$ most -1 . This then is the weakest possible bending at contact and might thus give rise to the slowest profile flattening.

Combining this with the results on the energy barrier we find that increasing the tension reduces the total penetration, and thereby flattens the profiles, but increases the energy stored in the membrane. This indicates that the dominant source of free membrane energy is tension, even though the reason why the profile is not simply flat is the curvature. Separating tension and bending contributions to the energy of the free membrane confirms this suspicion (not shown). 


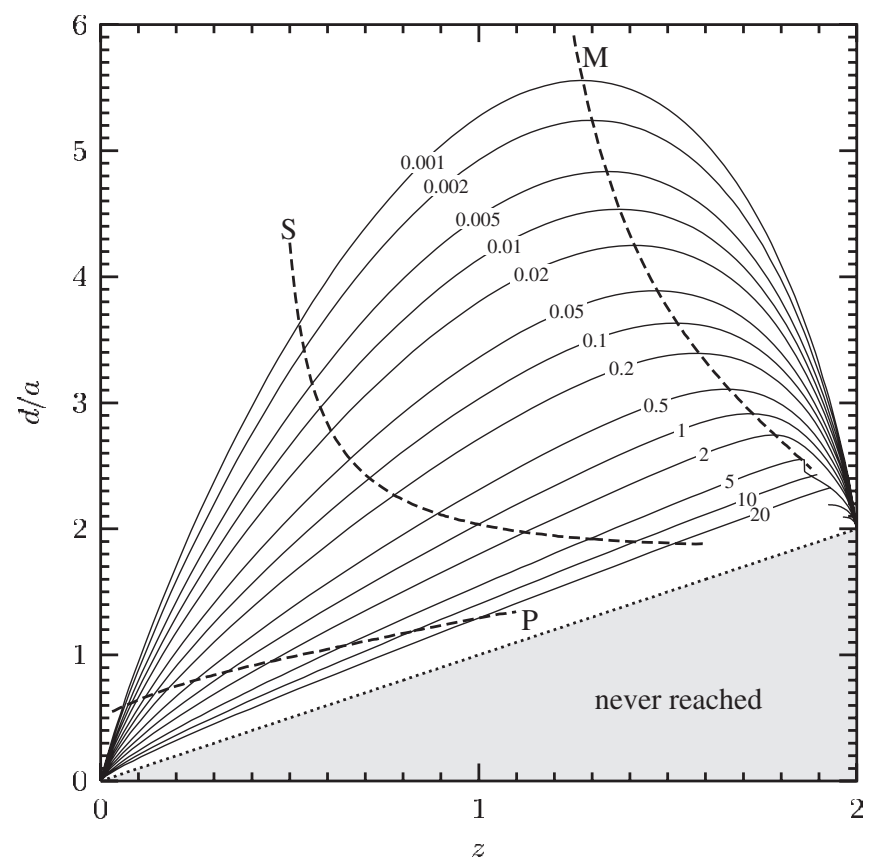

Figure 6. Total penetration $d$ (see text) as a function of the usual degree of penetration $z$ for various rescaled tensions $\tilde{\sigma}$ as indicated by the numbers. Lower tensions lead to higher total penetrations $d$. The line marked ' $\mathrm{P}$ ' indicates where the envelopment transition occurs. Line ' $\mathrm{S}$ ' indicates the spinodal. Line 'M' joins the maxima of all curves $d(z)$. The grey region below the dotted line $d / a=z$ is never reached, since the total membrane penetration is always at least as big as the detachment penetration $z$. For tensions somewhat larger than $\tilde{\sigma} \approx 4.7$ the high $z$ behaviour is complicated by a profile instability.

\section{Small gradient expansion}

If the membrane slope $\psi$ remains small (as is for instance the case for the small degrees of equilibrium wrapping occurring at low tension), one may expand the shape equations to lowest order in $\psi$. This approximation is very commonly used, for instance when calculating capillary interactions [23]. It is quickest done in the functional itself, but a derivation from the Hamilton equations themselves is also quite illustrative.

Equation (6e) shows that $\dot{p}_{r}$ is quadratic in the $\psi$ variables (counting $\dot{\psi}$ also of order $\psi)$, hence up to linear order $\dot{p}_{r}=0$ and together with equations $(6 f)$ and (10) we find that both $p_{r}$ and $p_{h}$ vanish identically. Next, in linear order $(6 a)$ becomes $r \dot{\psi}=\frac{1}{2} p_{\psi}-\psi$ and differentiation yields $r \ddot{\psi}=\frac{1}{2}\left(p_{\psi} / r+2 \tilde{\sigma} r \psi / a^{2}\right)-2 \dot{\psi}$ (since $\left.\dot{r}=1+\mathcal{O}\left(\psi^{2}\right)\right)$. Differentiating once again and inserting $(6 d)$ gives

$$
\dddot{\psi}+2 \frac{\ddot{\psi}}{r}-\left(\frac{1}{r^{2}}+\frac{\tilde{\sigma}}{a^{2}}\right) \dot{\psi}+\frac{1}{r}\left(\frac{1}{r^{2}}-\frac{\tilde{\sigma}}{a^{2}}\right) \psi=0 .
$$

Noting finally that in linear order $h^{\prime}:=\partial h / \partial r=\psi$ and $\partial / \partial r=\partial / \partial s$, we can rewrite (12) as $\Delta\left(\Delta-\lambda^{-2}\right) h(r)=0$, where $\Delta$ is the cylindrical Laplacian and $\lambda=\sqrt{\kappa / \sigma}$. The general solution of this linear differential equation is a linear combination of the eigenfunctions of the Laplacian with eigenvalues 0 and $\lambda^{-2}$, and the only two for which the profile does not diverge are a constant and the modified Bessel function $K_{0}$. Fixing the contact conditions (7) 
of smooth touching, the solution is readily found to be

$$
h(r)=h_{0}+\lambda \frac{r_{0}}{h_{0}} \frac{K_{0}(r / \lambda)-K_{0}\left(r_{0} / \lambda\right)}{K_{1}\left(r_{0} / \lambda\right)},
$$

where contact distance $r_{0}=a \sin \alpha$ and contact height $h_{0}=-a \cos \alpha$ have been used. An immediate result from this is the total penetration $d$ :

$$
\frac{d}{a}=1+\frac{h(\infty)}{a}=\left(1-\ln \frac{\tilde{\sigma} z}{E}\right) z+\mathcal{O}\left(z^{2}\right),
$$

where $E=2 \mathrm{e}^{-2 \gamma} \approx 0.6305$ and $\gamma=0.5772 \ldots$ is the Euler-Mascheroni constant. Inserting (13) back into the small gradient energy functional, performing the integrals, and expanding the result in powers of $z$, one finally gets [24]

$$
\tilde{E}_{\text {free }}=-2 \tilde{\sigma} z^{2} \ln \frac{\tilde{\sigma} z}{E}+\mathcal{O}\left(z^{3}\right)
$$

Equation (15) together with (2) yields the total energy of the wrapped complex in the small gradient approximation, and the equilibrium penetration again follows from $\partial \tilde{E} / \partial z=0$. Due to the logarithm in (15) this unfortunately cannot be expressed in terms of elementary functions, but one can give the following closed expression:

$$
z=-\frac{\tilde{w}-4}{4 \tilde{\sigma} W} \quad \text { with } W=W(\tilde{w})=\mathcal{W}_{-1}\left(-\frac{\tilde{w}-4}{4 E}\right),
$$

where $\mathcal{W}_{-1}$ is the branch -1 of the so-called Lambert $W$ function [25], which is the inverse of $x \mathrm{e}^{x}$ and which for small negative arguments behaves roughly like $\mathcal{W}_{-1}(x) \simeq \ln |x|-\ln |\ln | x||$. Incidentally, for adsorbing cylinders one also finds $z \propto \sigma^{-1}$, but the increase with $w$ beyond the onset of binding is quadratic, and $E_{\text {free }}(z) \propto z+\mathcal{O}\left(z^{2}\right)$ [26].

Together with the condition ${ }^{3} \tilde{E}(z)=\tilde{E}(2)=-2(\tilde{w}-4)+4 \tilde{\sigma}$ one obtains the envelopment boundary

$$
\frac{2 \tilde{\sigma}}{\tilde{w}-4}=\frac{1}{2}\left[1+\sqrt{1+\frac{1}{2 W}+\frac{1}{(2 W)^{2}}}\right] \stackrel{\tilde{w} \rightarrow^{4^{+}}}{{ }^{2}} 1+\left(8 \ln \frac{\tilde{w}-4}{4 E}\right)^{-1} .
$$

In particular, this also shows that $\tilde{w}=4+2 \tilde{\sigma}$ is the correct asymptotic behaviour in the limit $\tilde{\sigma} \rightarrow 0$ (in which $\tilde{w} \rightarrow 4$ ), and that the corrections are essentially logarithmic.

\section{Summary and conclusion}

The results of the previous two sections offer a clear picture of the adhesion and wrapping scenario: initially adhesion is only opposed by bending, tension energy setting in later to determine the degree of wrapping. At sufficient adhesion strength the colloid should become enveloped, but a substantial energy barrier gives rise to hysteresis. This holds even for most of the small tension regime $\tilde{\sigma} \leqslant 1$, because a tension energy small compared to bending need not be small compared to the thermal energy. Indeed, at $\tilde{\sigma}=1$ the tension energy scales as $E_{\text {ten }} \simeq \sigma a^{2}=\kappa$, and $\kappa$ is usually large compared to $k_{\mathrm{B}} T$. Increasing the tension can promote unwrapping, but not unbinding, which is independent of $\tilde{\sigma}$.

Since $\tilde{w}$ and $\tilde{\sigma}$ - the relevant variables of the structural phase diagram—can be controlled externally with relative ease, many quantitative experimental tests of the presented theory are possible. In order to avoid the small length scales it would then be advisable to focus on model

3 Note that here we use an exact result from the full shape equation (namely, the ideal neck theorem [22]), which cannot be derived within the small gradient framework. Also, it does not hold for wrapped cylinders. 
membranes with significantly larger bending constants, for instance membranes built from block-copolymers. It is also worth pointing out that tuning of these variables is very likely exploited by any biological system which relies on such wrapping events. For instance, in the case of viral budding the virus has various control possibilities over the adhesion strength: if electrostatic interactions play a role, changes in the $\mathrm{pH}$ will titrate the charges of the weak amino acids present in the capsid proteins. Moreover, if the virus synthesizes spikes for mediating the binding, their concentration in the plasma membrane of the host will determine the effective adhesion energy per unit area [7, 8]. Furthermore, the virus is, during its 'lifetime', in contact with various different membranes, at which it has to wrap or unwrap, and their tensions are not necessarily the same.

Let us finally remember that the above theory only studied the energetics of binding. Entropic aspects, most notably the influence of membrane fluctuations, have been left out. Their presence will most likely lead to an additional repulsion between colloid and membrane, the precise form of which will be the subject of future work.

\section{Acknowledgments}

I would like to thank T Bickel and W M Gelbart for fruitful discussions and collaborations. Financial support by the German Science Foundation under grant De775/1-2 is also gratefully acknowledged.

\section{References}

[1] Sackmann E 1996 Science 27143

[2] Garoff H, Hewson R and Opstelten D-J E 1998 Microbiol. Mol. Biol. Rev. 621171

[3] Sieczkarski S B and Whittaker G R 2002 J. Gen. Virol. 831535

[4] Garoff H and Simons K 1974 Proc. Natl Acad. Sci. USA 713988

[5] Simons K, Garoff H and Helenius A 1982 Sci. Am. 24658

[6] Lu Y E and Kielian M 2000 J. Virol. 747708

[7] van Effenterre D and Roux D 2003 Europhys. Lett. 64543

[8] Tzlil S, Deserno M, Gelbart W M and Ben-Shaul A 2004 Biophys. J. at press

[9] Boussif O, Lezoualc'h F, Zanta M A, Mergny M D, Scherman D, Demeneix B and Behr J-P 1995 Proc. Natl Acad. Sci. 927297

[10] Hochmuth R M, Shao J-Y, Dai J and Sheetz M P 1996 Biophys. J. 70358

[11] Abercrombie M, Heaysman J E M and Pegrum S M 1970 Exp. Cell Res. 62389

[12] Caspi A, Yeger O, Grosheva I, Bershadsky A D and Elbaum M 2001 Biophys. J. 811990

[13] Helfrich W 1973 Z. Naturf. c 28693

[14] Morris C E and Homann U 2001 J. Membr. Biol. 17979

[15] Seifert U and Lipowsky R 1995 Structure and Dynamics of Membranes (Handbook of Biological Physics) ed R Lipowsky and E Sackmann (Amsterdam: North-Holland)

[16] Lipowsky R and Döbereiner H-G 1998 Europhys. Lett. 43219

[17] Dinsmore A D, Wong D T, Nelson P and Yodh A G 1998 Phys. Rev. Lett. 80409

[18] Seifert U and Lipowsky R 1990 Phys. Rev. A 424768

[19] Capovilla R and Guven J 2002 Phys. Rev. E 66041604

[20] Deserno M 2004 Phys. Rev. E at press (see also Deserno M 2003 Preprint cond-mat/0303656)

[21] Courant R and Hilbert D 1953 Methods of Mathematical Physics vol 1 (New York: Interscience)

[22] Fourcade B, Miao L, Rao M and Wortis M 1994 Phys. Rev. E 495276

[23] Kralchevsky P A and Nagayama K 2000 Adv. Colloid Interface Sci. 85145

[24] Deserno M and Bickel T 2003 Europhys. Lett. 62767

[25] Corless R M, Gonnet G H, Hare D E G, Jeffrey D J and Knuth D E 1996 Adv. Comput. Math. 5329

[26] Weikl T R 2003 Eur. Phys. J. E 12265 